\title{
OPTIMAL SMOOTHING FOR CONVEX POLYTOPES
}

\author{
MOHAMMAD GHOMI
}

\begin{abstract}
It is proved that given a convex polytope $P$ in $\mathbf{R}^{n}$, together with a collection of compact convex subsets in the interior of each facet of $P$, there exists a smooth convex body arbitrarily close to $P$ which coincides with each facet precisely along the prescribed sets, and has positive curvature elsewhere.
\end{abstract}

\section{INTRODUCTION}

It has been known since the foundational work of H. Minkowski [9], see [1, p. 39], that the boundary of every convex polytope $P$ in Euclidean space $\mathbf{R}^{n}$ may be approximated, in the sense of Hausdorff distance, by an analytic convex hypersurface. There have been also some refinements of this theorem due to P. Hammer [7] and W. Firey [3] who extended it to algebraic hypersurfaces. Though these approximations are as smooth as one could wish, for certain purposes they may have a drawback: they do not coincide with $P$ along any open subset. Thus in this paper we are led to develop a smoothing procedure which preserves $P$ along prescribed regions:

Theorem 1.1. Let $P \subset \mathbf{R}^{n}$ be a convex polytope, with interior points, and facets $F_{i}, i=1, \ldots, k$. Let $X_{i}$ be a compact convex subset in the interior of $F_{i}$. Then for every $\epsilon>0$ there exists a convex body $K \subset P$ with smooth $\left(C^{\infty}\right)$ boundary $\partial K$ such that

1. $\partial K \cap F_{i}=X_{i}$,

2. $\partial K-\cup_{i} X_{i}$ has positive curvature,

3. $\operatorname{dist}(K, P) \leq \epsilon$.

where dist denotes Hausdorff distance. Furthermore, if $\cup_{i} X_{i}$ is symmetric with respect to some rigid motion in $\mathbf{R}^{n}$, then there exists a convex body $K$, satisfying the above properties, which has the same symmetry.

The above smoothing may be considered "optimal" in the sense that it preserves the boundary of $P$ precisely as much or as little as desired. In the case where each $X_{i}$ is a point, the above has been proved by W. Weil [12], using a certain convolution first devised by C. Berg, and further studied by R. Schneider $[11,10]$. Our proof also employs this convolution together with some recent

1991 Mathematics Subject Classification. 53A07, 52B11, 53C45.

Key words and phrases. Smooth approximation, convex polytopes, support function, convolution, Gaussian curvature.

The author was partially supported by the NSF grant DMS-0204190, and CAREER award DMS-0332333. 
results on strictly convex submanifolds [4]. The above may be of interest in studying Brownian motion in convex polygons [8], constructing "subsolutions" for Monge-Ampére equations [4], smoothing of convex functions [5], and approximating general convex bodies [6]. The above theorem improves [4, Thm 1.2.4], where a similar smoothing had been constructed under the additional requirement that $X_{i}$ is smooth and has positively curved boundary.

By a convex body $K \subset \mathbf{R}^{n}$ we mean a compact convex set with interior points. A polytope $P \subset \mathbf{R}^{n}$ is a convex body which is the intersection of finitely many closed half-spaces. A facet $F_{i}$ of $P$ is the intersection of $P$ with a support hyperplane $H_{i}$ provided that $F_{i}$ has interior points in $H_{i}$. By smooth we always mean differentiable of class $C^{\infty}$. A point $p$ in the boundary $\partial K$ is a smooth point if an open neighborhood of $p$ in $\partial K$ admits a $C^{\infty}$ parametrization, e.g., it is the graph of a $C^{\infty}$ (convex) function over a support hyperplane of $K$ at $p$. If this function has positive definite hessian, then we say that $K$ has positive curvature at $p$.

Note 1.2. It is easy to satisfy property 1 of Theorem 1.1, if we require that $\partial K$ be only differentiable of class $C^{1,1}$. To see this let $\nu_{i}$ be the outward unit normal to the facet $F_{i}, \delta>0$, and $X_{i}^{\delta}:=X_{i}-\delta \nu_{i}$ be the translation of $X_{i}$ into $P$. Let $\bar{P}:=\operatorname{conv}\left(\cup_{i} X_{i}^{\delta}\right)$ be the convex hull of these translations. An elementary computation shows that if

$$
\delta<\inf \left\{\frac{\left\langle x_{j}-x_{i}, \nu_{j}\right\rangle}{1-\left\langle\nu_{i}, \nu_{j}\right\rangle}: x_{i} \in X_{i}, x_{j} \in X_{j}, i \neq j\right\},
$$

then $\cup_{i} X_{i}^{\delta} \subset \partial \bar{P}$, where $\langle\cdot, \cdot\rangle$ denotes the standard inner product in $\mathbf{R}^{n}$. Consequently $K:=\bar{P}+\delta B^{n}$, the outer parallel body of $\bar{P}$ at the distance $\delta$, is the desired object ( $B^{n}$ denotes the unit ball in $\mathbf{R}^{n}$ ).

Note 1.3. Proving Theorem 1.1 is not difficult if we weaken condition 1 to $X_{i} \subset K \cap F_{i}$, and disregard condition 2. To see this suppose that $P$ contains the origin of $\mathbf{R}^{n}$ in its interior, and let $\rho: \mathbf{R}^{n} \rightarrow \mathbf{R}$, given by

$$
\rho(x):=\inf \{\lambda>0: x \in \lambda P\},
$$

be the distance function of $P$. Then $\rho$ is a convex piecewise linear function with $\rho^{-1}([0,1])=P$. Let $\widetilde{\rho}$ be the convolution of $\rho$ with a positive and centrally symmetric approximate identity function $\theta_{\epsilon}: \mathbf{R}^{n} \rightarrow \mathbf{R}$ with support inside a ball of radius $\epsilon$. Choose $\epsilon$ sufficiently small so that an $\epsilon$-neighborhood of $X_{i}$, in the affine hull of $F_{i}$, lies in $F_{i}$. Then $K:=\widetilde{\rho}^{-1}([0,1])$ is the desired body; because, the convolution preserves convexity and fixes $\rho$ over any compact subset of an open region where $\rho$ is linear.

To prove Theorem 1.1 we require a pair of propositions which are proved in the next two sections.

\section{Smooth Convex Functions with Prescribed Minima}

We say a $C^{2}$ convex function $f: \mathbf{R}^{n} \rightarrow \mathbf{R}$ is strictly convex on a subset $U \subset \mathbf{R}^{n}$ if the Hessian of $f$ is positive definite on $U$. Recall that, for every $p \in U$, Hess $f_{p}$ 
is the bilinear form on $\mathbf{R}^{n} \times \mathbf{R}^{n}$ given by

$$
\text { Hess } f_{p}(v, w):=\sum_{i, j=1}^{n} D_{i j} f(p) v_{i} w_{j} .
$$

Note that if $f$ has positive definite hessian, then the graph of $f$ contains no line segments. Thus our definition of strict convexity is stronger than the one which is commonly used in convexity texts.

Proposition 2.1. For every compact convex subset $X \subset \mathbf{R}^{n}$, there exists a smooth nonnegative convex function $f: \mathbf{R}^{n} \rightarrow \mathbf{R}$ such that $f^{-1}(\{0\})=X$, and $f$ is strictly convex on $\mathbf{R}^{n}-X$.

Proof. After a translation, we may assume that the origin $o$ of $\mathbf{R}^{n}$ is contained in $X$. Let $h: \mathbf{R}^{n} \rightarrow \mathbf{R}$ be the support function of $X$, that is

$$
h(\cdot):=\sup _{x \in X}\langle x, \cdot\rangle .
$$

Note that, for every $u$ in the sphere $\mathbf{S}^{n-1}, h(u)$ is the distance between $o$ and the support hyperplane

$$
H_{u}:=\left\{p \in \mathbf{R}^{n}:\langle p, u\rangle=h(u)\right\} .
$$

Let $g: \mathbf{R} \rightarrow \mathbf{R}$ be any smooth function which is strictly convex on $(0, \infty)$, but vanishes on $(-\infty, 0]$. For instance, we may set:

$$
g(x):= \begin{cases}x^{2} \exp \left(\frac{-1}{x^{2}}\right), & \text { if } x>0 \\ 0, & \text { otherwise. }\end{cases}
$$

Define $\phi: \mathbf{S}^{n-1} \times \mathbf{R}^{n} \rightarrow \mathbf{R}$ by

$$
\phi(u, p):= \begin{cases}g(\langle p, u\rangle-h(u)), & \text { if }\langle p, u\rangle>h(u) ; \\ 0, & \text { otherwise. }\end{cases}
$$

Thus, for every $u \in \mathbf{S}^{n-1}, \phi(u, \cdot)$ is a smooth convex function which vanishes on $X$, but is positive in the half space $\langle p, u\rangle>h(u)$. Set

$$
f(p):=\int_{\mathbf{S}^{n-1}} \phi(u, p) d u .
$$

Since $\phi$ is smooth, $f$ is smooth, and one easily verifies that it is convex as well, using the linearity of integrals. Further, it is clear that $f$ vanishes on $X$. On the other hand, if $p \notin X$, then there exists a support hyperplane $H_{u_{0}}$ which separates $p$ and $X$, because $X$ is convex. Thus, $\phi(u, p)>0$ for all $u$ in a neighborhood of $u_{0}$. Since $\phi \geq 0$ everywhere, this yields that $f(p)>0$. So $f$ vanishes precisely on $X$.

It remains to check that the Hessian of $f$ is positive definite on $\mathbf{R}^{n}-X$. To this end recall that

$$
\operatorname{Hess} f_{p}(v, v)=\left.\frac{d^{2}}{d t^{2}} f(p+t v)\right|_{t=0} .
$$


Next note that $t \mapsto \phi(u, p+t v)$ is convex. Thus, $d^{2} \phi(u, p+t v) / d t^{2} \geq 0$, which yields that, for every $p, v \in \mathbf{R}^{n}$ and $U \subset \mathbf{S}^{n-1}$,

$$
\frac{d^{2}}{d t^{2}} f(p+t v)=\int_{\mathbf{S}^{n-1}} \frac{d^{2}}{d t^{2}} \phi(u, p+t v) d u \geq \int_{U} \frac{d^{2}}{d t^{2}} \phi(u, p+t v) d u .
$$

For each $p \in \mathbf{R}^{n}-X$ there exists a $u_{p} \in \mathbf{S}^{n-1}$ such that $H_{u_{p}}$ separates $p$ and $X$. Then $\left\langle p, u_{p}\right\rangle>h\left(u_{p}\right)$. So there exists an open neighborhood $U_{p} \subset \mathbf{S}^{n-1}$ and an $\epsilon_{p}>0$ such that for all $(u, t) \in U_{p} \times\left(-\epsilon_{p}, \epsilon_{p}\right)$, and $v \in \mathbf{S}^{n-1},\langle p+t v, u\rangle>h(u)$. Consequently, for these values, the definition of $\phi$ yields that

$$
\phi(u, p+t v)=g(\langle p+t v, u\rangle-h(u)) .
$$

When $\langle p+t v, u\rangle-h(u)>0$, the above is strictly convex in $t$, in which case

$$
\left.\frac{d^{2}}{d t^{2}} \phi(u, p+t v)\right|_{t=0}>0
$$

Thus in (5) if we set $U:=U_{p}$, then $d^{2} f(p+t v) /\left.d t^{2}\right|_{t=0}>0$, for all $p \in \mathbf{R}^{n}-X$ and $v \in \mathbf{S}^{n-1}$. So by (4) Hess $f_{p}$ is positive definite on $\mathbf{R}^{n}-X$.

Note 2.2. For $\epsilon>0$, let $X_{\epsilon}:=f^{-1}([0, \epsilon])$, where $f$ is as in (3). This yields a family of convex bodies with smooth boundary which, as $\epsilon \rightarrow 0$, converges to $X$ in the sense of Hausdorff distance.

\section{Completion of Strictly Convex Patches}

Recall that the support function of a convex body, as defined by (2), is a convex and positively homogeneous function $h: \mathbf{R}^{n} \rightarrow \mathbf{R}$. Conversely, every such function uniquely determines a convex body

$$
K=\left\{x \in \mathbf{R}^{n}:\langle x, p\rangle \leq h(p), \text { for all } p \in \mathbf{R}^{n}\right\},
$$

[11, Thm. 1.7.1]. We say $v \in \mathbf{S}^{n-1}$ is a support vector for $p \in \partial K$, if $K$ lies on one side of the support hyperplane $H$ which is orthogonal to $v$ and passes through $p$. Further, if $p+v$ lies in the halfspace of $H$ not containing $K$, then we say that $v$ is an outward support vector. When $p$ is a smooth point of $\partial K$, the (unique) support hyperplane of $K$ at $p$ is denoted by $T_{p} \partial K$, and is called the tangent hyperplane of $K$ at $p$.

Lemma 3.1. Let $K \subset \mathbf{R}^{n}$ be a convex body with support function $h$, and $v_{0} \in$ $\mathbf{S}^{n-1}$ be an outward support vector for $p_{0} \in \partial K$. Then the following are equivalent:

1. $p_{0}$ is a smooth point of $\partial K$, and $\partial K$ has positive curvature at $p_{0}$.

2. $v_{0}$ is a smooth point of $h$, and $h$ is strictly convex on $T_{v_{0}} \mathbf{S}^{n-1}$.

Though the above is essentially known, e.g. see [11, p. 103-109], we include a concise proof for lack of an explicit reference.

Proof. $(\mathbf{1} \Rightarrow \mathbf{2})$. Let $U \subset \partial K$ be an open neighborhood of $p_{0}$ which is smooth and positively curved. Then the inverse function theorem implies that the outward 
unit normal, or the Gauss map, $\nu: U \rightarrow \nu(U) \subset \mathbf{S}^{n-1}$, is a diffeomorphism. Consequently, setting $V:=\nu(U)$, we obtain a one-to-one correspondence

$$
\partial K \supset U \ni p \longleftrightarrow v \in V \subset \mathbf{S}^{2} .
$$

In particular, using the above convention, we may write

$$
h(v)=\langle p, v\rangle .
$$

Thus $\left.h\right|_{V}$ is smooth, which, since $h$ is homogeneous, yields that $h$ is smooth on (an open neighborhood of) $V$. Further, the above equation yields that the gradient of $h$ on $V$ is given by

$$
\operatorname{grad} h(v):=\left(D_{1} h(v), \ldots, D_{n} h(v)\right)=p .
$$

It is a basic fact in differential geometry that, since $\partial K$ has positive curvature on $U$, for every $p \in U$ there exists a basis $e_{i}=e_{i}(p), 1 \leq i \leq n-1$, for the tangent hyperplane $T_{p} \partial K$ such that

$$
d \nu_{p}\left(e_{i}\right)=k_{i} e_{i}
$$

where $d$ is the differential map, and $k_{i}=k_{i}(p)>0\left(e_{i}\right.$ are the "principle directions" and $k_{i}$ are the corresponding "principal curvatures").

Note that $T_{p} \partial K$ is parallel to $T_{v} \mathbf{S}^{n-1}$. Thus $\left\{e_{i}\right\}$ also forms a basis for $T_{v} \mathbf{S}^{n-1}$, and using the last two equations above, we have

$$
\text { Hess } h_{v}\left(e_{i}, e_{j}\right)=\left\langle D_{e_{i}} \operatorname{grad} h(v), e_{j}\right\rangle=\left\langle d \nu_{v}^{-1}\left(e_{i}\right), e_{j}\right\rangle= \begin{cases}\frac{1}{k_{i}}, & \text { if } i=j ; \\ 0, & \text { otherwise. }\end{cases}
$$

So we conclude that $h$ is strictly convex on $T_{v} \mathbf{S}^{n-1}$.

$(\mathbf{2} \Rightarrow \mathbf{1})$ Let $V \subset \mathbf{S}^{n-1}$ be an open neighborhood of $v_{0}$ where $h$ is smooth and strictly convex on $T_{v} \mathbf{S}^{n-1}$ for all $v \in V$. Define $f: V \rightarrow \mathbf{R}^{n}$ by

$$
f(v):=\operatorname{grad} h_{v} .
$$

Since the restriction of Hess $h_{v}$ to $T_{v} \mathbf{S}^{n-1}$ is positive definite, for every nonzero vector $x \in T_{v} \mathbf{S}^{n-1}$ we have

$$
\left\langle d f_{v}(x), x\right\rangle=\left\langle D_{x} \operatorname{grad} h(v), x\right\rangle=\operatorname{Hess} h_{v}(x, x)>0 .
$$

So $d f_{v}$ is nondegenerate which yields that $f: V \rightarrow f(V) \subset \partial K$ is a diffeomorphism, assuming $V$ is sufficiently small. In particular, $U:=f(V)$ is a smooth open subset of $\partial K$. Now define $\nu: U \rightarrow \mathbf{S}^{n-1}$ by $\nu(f(v))=v$. For all $v \in V$, and $x \in T_{v} \mathbf{S}^{n-1}$,

$$
\left\langle d f_{v}(x), v\right\rangle=\left\langle x, D_{v} \operatorname{grad} h(v)\right\rangle=0
$$

because, since $h$ is homogenous, $D_{v} \operatorname{grad} h(v)=0$. So $v$ is orthogonal to $T_{f(v)} \partial K$, which yields that $\nu$ is the Gauss map of $U$. Since $\nu \circ f$ is the identity, and $d f_{v_{0}}$ is nondegenerate, it follows that $d \nu_{p_{0}}=\left(d f_{v_{0}}\right)^{-1}$. So the eigenvalues of $d \nu_{p_{0}}$ are reciprocal of those of $d f_{v_{0}}$, which are positive by (6). So $\partial K$ has positive curvature at $p_{0}$. 
Let $K \subset \mathbf{R}^{n}$ be a convex body with support function $h$. For $\epsilon>0$, let $\theta_{\epsilon}:[0, \infty) \rightarrow[0, \infty)$ be a smooth function with $\operatorname{support} \operatorname{supp}\left(\theta_{\epsilon}\right) \subset[\epsilon / 2, \epsilon]$, $\int_{\mathbf{R}^{n}} \theta_{\epsilon}(\|x\|) d x=1$, and set

$$
\widetilde{h^{\epsilon}}(p):=\int_{\mathbf{R}^{n}} h(p+\|p\| x) \theta_{\epsilon}(\|x\|) d x,
$$

where $\|\cdot\|:=\langle\cdot, \cdot\rangle^{\frac{1}{2}}$ denotes the standard norm in $\mathbf{R}^{n}$. It is not difficult to show that $\widetilde{h^{\epsilon}}$ is convex and positively homogeneous; thus it determines a convex body $\widetilde{K^{\epsilon}}$ which we call the Schneider transform of $K[11$, p. 158]. We say that the radii of curvature of $K$ are bounded below if there exists an $r>0$ such that through every point $p \in \partial K$ there passes a ball $B$ of radius $r$ contained inside $K$ (one may also say that $B$ "rolls freely" inside $K$ ).

The following lemma is also known, but again a proof is included because the author is not aware of an explicit reference.

Lemma 3.2. Let $K \subset \mathbf{R}^{n}$ be a convex body whose radii of curvature are bounded below. Then the Schneider transform of $K$ is smooth, and has positive curvature.

Proof. Suppose that the radii of curvature of $K$ are bounded below by $r$. Set

$$
L:=\left\{p \in K: B^{n}(p, r) \subset K\right\},
$$

where $B^{n}(p, r)$ denotes the ball of radius $r$ centered at $p$. Then $L$ is a convex body, and $K=L+B^{n}(o, r)$, where + denotes Minkowski addition. So, $h_{K}=$ $h_{L}+h_{B^{n}(o, r)}$, which in turn yields

$$
\widetilde{h}^{\epsilon}{ }_{K}(u)=\widetilde{h}^{\epsilon} L(u)+\widetilde{h}^{\epsilon}{ }_{B^{n}(o, r)}(u)=\widetilde{h}^{\epsilon}(u)+r\|u\| .
$$

Note that the restriction of $\|\cdot\|$ to $T_{p} \mathbf{S}^{n-1}$ is strictly convex, for all $p \in \mathbf{S}^{n-1}$. Thus $\widetilde{h^{\epsilon}}{ }_{K}$ is strictly convex on the tangent hyperplanes of the sphere, which, by Lemma 3.1, yields that $\widetilde{K}$ is smooth and has positive curvature.

We say a smooth hypersurface $M \subset \mathbf{R}^{n}$ is strictly convex if, for all $p \in M$, (i) $M$ lies on one side the tangent hyperplane $T_{p} M$, (ii) $M \cap T_{p} M=\{p\}$, and (iii) $M$ has positive curvature at $p$. Unless stated otherwise, our hypersurfaces may be disconnected and may have boundary.

Proposition 3.3. Let $\widetilde{M} \subset \mathbf{R}^{n}$ be a smooth strictly convex hypersurface without boundary, and $M \subset \widetilde{M}$ be compact. Then $M$ lies on the boundary of a smooth convex body with positive curvature.

The above is a special case of the main result of [4]. Since the special case may be treated much more concisely, however, we include a proof:

Proof. Let $U \subset \widetilde{M}$ be an open subset with compact closure $\bar{U}$, and $U \supset M$. Let $\nu: \widetilde{M} \rightarrow \mathbf{S}^{n-1}$ be the Gauss map, and, for small $r>0$, define the inner parallel hypersurface of $\bar{U}$ by

$$
\bar{U}_{r}:=\left\{p_{r}:=p-r \nu(p): p \in \bar{U}\right\} .
$$


Since the curvature of $\bar{U}_{r}$ depends continuously on $r$, and $\bar{U}$ is compact, $\bar{U}_{r}$ has positive curvature (for $r$ sufficiently small). Thus $\bar{U}_{r}$ lies locally on one side of each of its tangent hyperplanes. Equivalently, if we define $f_{r}: \bar{U} \times \bar{U} \rightarrow \mathbf{R}$ as

$$
f_{r}(p, q):=\left\langle p_{r}-q_{r}, \nu\left(q_{r}\right)\right\rangle,
$$

the signed distance between $p_{r}$ and $T_{q_{r}} \bar{U}$, then $f_{r} \leq 0$ on an open neighborhood $A$ of the diagonal of $\bar{U} \times \bar{U}$. Since by assumption $\bar{U}$ is strictly convex, $f_{0}<0$ on $B:=\bar{U} \times \bar{U}-A$. So, since $B$ is compact, it follows that $f_{r}<0$ on $B$ as well. Consequently $\bar{U}_{r}$ lies globally on each side of its tangent hyperplanes, or, equivalently, $\bar{U}_{r} \subset \partial \operatorname{conv}\left(\bar{U}_{r}\right)$. Thus setting

$$
K:=\operatorname{conv}\left(\bar{U}_{r}\right)+B^{n}(o, r),
$$

we obtain a convex body with $\bar{U} \subset \partial K$.

Let $V \subset U$ be an open set with $M \subset V$ and $\bar{V} \subset U$. Set $U^{\prime}:=\nu(U)$, and $V^{\prime}:=\nu(V)$. Then $U^{\prime}$ and $V^{\prime}$ are open in $\mathbf{S}^{n-1}$, because, since the curvature of $U$ is nonzero, $\nu$ is a local diffeomorphism. Let $\bar{\phi}: \mathbf{S}^{n-1} \rightarrow \mathbf{R}$ be a smooth function with support $\operatorname{supp}(\bar{\phi}) \subset U^{\prime}$, and $\left.\bar{\phi}\right|_{\bar{V}^{\prime}} \equiv 1$. Let $\phi$ be the extension of $\bar{\phi}$ to $\mathbf{R}^{n}$ given by $\phi(o):=0$, and $\phi(p):=\bar{\phi}(p /\|p\|)$, when $p \neq o$. Define $\bar{h}: \mathbf{R}^{n} \rightarrow \mathbf{R}$ by

$$
\bar{h}^{\epsilon}(p):=\widetilde{h}^{\epsilon}(p)+\phi(p)\left(h(p)-\widetilde{h^{\epsilon}}(p)\right),
$$

where $h$ is the support function of $K$ and $\widetilde{h^{\epsilon}}$ is as in (7). We claim that there exists an $\epsilon>0$, giving an $\bar{h}^{\epsilon}$ such that

$$
\bar{K}^{\epsilon}:=\left\{x \in \mathbf{R}^{n}:\langle x, p\rangle \leq \bar{h}^{\epsilon}(p) \text {, for all } p \in \mathbf{R}^{n}\right\}
$$

is the desired body.

To establish the above claim, with an eye towards applying Lemmas 3.1 and 3.2 , we first show that $\bar{K}^{\epsilon}$ is a convex body with support function $\bar{h}^{\epsilon}$. To this end, it suffices to check that $\bar{h}^{\epsilon}$ is positively homogeneous and convex. Homogeneity of $\bar{h}^{\epsilon}$ is immediate from the definition. Thus to see convexity, it suffices to show that Hess $\bar{h}_{p}^{\epsilon}$ is nonnegative semidefinite for all $p \in \mathbf{S}^{n-1}$. Since $\left.\bar{h}^{\epsilon}\right|_{\mathbf{S}^{n}-U^{\prime}}=\widetilde{h^{\epsilon}}$, and $\widetilde{h^{\epsilon}}$ is convex, we need to check this only for $p \in U^{\prime}$. To this end, note that, for each $p \in \bar{U}^{\prime},\left.h\right|_{T_{p} \mathbf{S}^{n-1}}$ is strictly convex. Further, by construction,

$$
\left\|h-\bar{h}^{\epsilon}\right\|_{C^{2}\left(\bar{U}^{\prime}\right)} \rightarrow 0,
$$

as $\epsilon \rightarrow 0$. So, for every $p \in \bar{U}^{\prime}$, there exists an $\epsilon(p)>0$ such that $\left.\bar{h}^{\epsilon}\right|_{T_{p} \mathbf{S}^{n-1}}$ is strictly convex. Since $\bar{U}^{\prime}$ is compact and $\epsilon(p)$ depends on the size of the eigenvalues of the Hessian matrix of $\left.\bar{h}^{\epsilon}\right|_{T_{p} \mathbf{S}^{n-1}}$, which in turn depend continuously on $p$, it follows that there is an $\epsilon>0$ such that $\left.\bar{h}^{\epsilon}\right|_{T_{p} \mathbf{S}^{n-1}}$ is strictly convex for all $p \in \bar{U}^{\prime}$. Next we show that $\partial K$ is smooth and positively curved. To this end, by Lemma 3.1, we need to check that $\left.\bar{h}^{\epsilon}\right|_{T_{p} \mathbf{S}^{n-1}}$ is strictly convex for all $p \in \mathbf{S}^{n-1}$. For $p \in U^{\prime}$, this was verified above. For $p \in \mathbf{S}^{n-1}-U^{\prime}$, note that $\bar{h}^{\epsilon}=\widetilde{h}^{\epsilon}$ on the cone spanned by $\mathbf{S}^{n-1}-U^{\prime}$. So it is enough to check that $\left.\widetilde{h}^{\epsilon}\right|_{T_{p} \mathbf{S}^{n-1}}$ is strictly 
convex. By Lemmas 3.2 and 3.1, this follows from the boundedness of the radii of curvature from below.

Finally, it remains to show that $M \subset \partial \bar{K}^{\epsilon}$. Since $M \subset U$, which is smooth in $\partial K$, we have $h(p)=\left\langle\nu^{-1}(p), p\right\rangle$, for all $p \in U^{\prime}$. Consequently $\operatorname{grad} h(p)=\nu^{-1}(p)$. Thus

$$
\nu^{-1}(p)=\operatorname{grad} h(p)=\operatorname{grad} \bar{h}^{\epsilon}(p)=\bar{\nu}^{-1}(p),
$$

where $\bar{\nu}$ is the Gauss map of $\bar{K}^{\epsilon}$. So $M \subset \bar{\nu}^{-1}\left(U^{\prime}\right) \subset \partial \bar{K}^{\epsilon}$.

\section{Proof of TheOrem 1.1}

By Proposition 2.1, for every facet $F_{i}$ of $P$ there exists a smooth convex function $f_{i}: F_{i} \rightarrow \mathbf{R}$ with $f_{i}^{-1}(\{0\})=X_{i}$. Let $\nu_{i}$ be the outward unit normal of $P$ at $F_{i}$ and set

$$
\text { Plate }_{i}:=\left\{p-f_{i}(p) \nu_{i}: p \in U_{\delta}\left(X_{i}\right)\right\},
$$

where $U_{\delta}\left(X_{i}\right)$ is a $\delta$-neighborhood of $X_{i}$ in the affine hull aff $\left(F_{i}\right)$, i.e., the hyperplane in $\mathbf{R}^{n}$ which contains $F_{i}$.. Set

$$
\text { Plates }:=\cup_{i} \text { Plate }_{i} .
$$

Since by assumption $X_{j}$ lies in the relative interior of $F_{j}$, we may choose $\delta>0$ small enough so that

$$
\operatorname{aff}\left(F_{i}\right) \cap \text { Plate }_{j}=\emptyset,
$$

for all $i \neq j$. Now define $d_{i}:$ Plate $_{i} \rightarrow \mathbf{R}$ by

$$
d_{i}(p):=\inf \left\{|\langle x-p, \nu(p)\rangle|: x \in\left(\text { Plates }- \text { Plate }_{i}\right)\right\},
$$

where $\nu$ : Plates $\rightarrow \mathbf{S}^{n-1}$ is the outward unit normal. Note that $d_{i}(p)$ is the distance between $T_{p}$ Plate $_{i}$ and Plates - Plate $_{i}$. Further, if $p \in X_{i}$, then $T_{p}$ Plate $_{i}=$ $\operatorname{aff}\left(F_{i}\right)$. Thus (8) implies $d_{i}>0$ on $X_{i}$. So, since $d_{i}$ is continuous and $X_{i}$ is compact, there exists $\delta_{i}>0$ such that $d_{i}>0$ on $U_{\delta_{i}}\left(X_{i}\right)$. Set $\delta:=\min _{i} \delta_{i}$. Then Plates lies on one side of each of its tangent hyperplanes; or, equivalently, it lies on the boundary of its own convex hull:

$$
\text { Plates } \subset \partial(\text { conv Plates }),
$$

where we also use the fact that each Plate ${ }_{i}$ is a convex hypersurface. Next define

$$
\operatorname{Rim}_{i}:=\left\{p-f_{i}(p) \nu_{i}: p \in U_{\delta}\left(X_{i}\right)-U_{\delta / 2}\left(X_{i}\right)\right\},
$$

and set

$$
\operatorname{Rims}:=\cup_{i} \operatorname{Rim}_{i} .
$$

Since $f_{i}$ has positive definite Hessian on $F_{i}-X_{i}$, it follows from (9) that Rims is a strictly convex hypersurface. Thus, by Proposition 3.3, Rims lies on the boundary of a smooth convex body $L \subset \mathbf{R}^{n}$ with positive curvature.

Let $\Gamma_{i}^{1}$ and $\Gamma_{i}^{2}$ be the boundary components of $\operatorname{Rim}_{i}$, i.e., the graphs over $\partial\left(U_{\delta}\left(X_{i}\right)\right)$ and $\partial\left(U_{\delta / 2}\left(X_{i}\right)\right)$ respectively. Note that since $U_{\delta}\left(X_{i}\right)$ is a convex body in $F_{i}, \Gamma_{i}^{1}$ is homeomorphic to $\mathbf{S}^{n-2}$. Thus, since $\partial L$ is homeomorphic to $\mathbf{S}^{n-1}$, it follows from the Jordan-Brouwer separation theorem that $\partial L-\Gamma_{i}^{1}$ has precisely 
two (connected) components. Let $C_{i}$ be the component of $\partial L-\Gamma_{i}^{1}$ which contains $\Gamma_{i}^{2}$. Set

$$
C:=\partial L-\cup_{i} C_{i} .
$$

Since each $C_{i}$ is topologically a disk, and $C_{i} \cap C_{j}=\emptyset$, whenever $i \neq j$, it follows that $C$ is connected. Further note that by construction $\partial X=\partial$ Plates, and the interior of $X$ is disjoint from Rims. Thus Plates $\cup C$ is a smooth closed hypersurface with nonnegative curvature. It follows then from a theorem of Chern and Lashof [2, Thm. 4] that Plates $\cup C$ bounds a convex body $K$. Further, by construction, $K \cap F_{i}=X_{i}$, and $\partial K-\cup_{i} X_{i}$ has positive curvature.

To push $K$ within an $\epsilon$ distance of $P$, choose in the interior of each $F_{i}$ a compact convex subset $Y_{i}$ such that $X_{i} \subset Y_{i}$. By the above construction, there exists then a smooth convex body $\bar{K}$ with $Y_{i} \subset \partial \bar{K}$. Choosing $Y_{i}$ sufficiently large, we may assume that $\operatorname{dist}(\bar{K}, P) \leq \epsilon / 2$. Suppose that $o \in \operatorname{int} \bar{K}$ and let $\bar{\rho}, \rho$ be the distance functions of $\bar{K}$ and $K$ respectively, as defined by (1). For $\lambda \in[0,1)$, set

$$
\rho_{\lambda}:=\lambda \bar{\rho}+(1-\lambda) \rho .
$$

Then $K_{\lambda}:=\rho_{\lambda}^{-1}([0,1])$ is a smooth convex body, because $\bar{\rho}$ and $\rho$ are both smooth convex functions. Further note that since $\rho, \bar{\rho} \geq 1$ on $F_{i}$, it follows that $\rho_{\lambda}(x)=1$ at $x \in F_{i}$, if and only if $\rho(x)=1=\bar{\rho}(x)$. Consequently

$$
\partial K_{\lambda} \cap F_{i}=\left(\partial \bar{K} \cap F_{i}\right) \cap\left(\partial K \cap F_{i}\right)=Y_{i} \cap X_{i}=X_{i} .
$$

Next we check that $\partial K_{\lambda}$ has positive curvature in the complement of $X:=\cup_{i} X_{i}$. Let $\nu$ be the Gauss map of $K_{\lambda}$. Since $\partial K_{\lambda}$ is a level set of $\rho_{\lambda}$, for every $e_{i}$, $e_{j} \in T_{p} \partial K_{\lambda}$ we have

$$
\left\langle d \nu_{p}\left(e_{i}\right), e_{j}\right\rangle=\left\langle D_{e_{i}} \frac{\operatorname{grad}\left(\rho_{\lambda}\right)_{p}}{\left\|\operatorname{grad}\left(\rho_{\lambda}\right)_{p}\right\|}, e_{j}\right\rangle=\frac{1}{\left\|\operatorname{grad}\left(\rho_{\lambda}\right)_{p}\right\|} \operatorname{Hess}\left(\rho_{\lambda}\right)_{p}\left(e_{i}, e_{j}\right) .
$$

Thus $\partial K_{\lambda}$ is positively curved at $p$, if and only if $\rho_{\lambda}$ is strictly convex on $T_{p} \partial K_{\lambda}$. Since $\rho_{\lambda}$ is homogeneous, this is equivalent to $\rho_{\lambda}$ being strictly convex on $T_{\nu(p)} \mathbf{S}^{n-1}$. If $p \notin X$, then the point on $K$ with outward normal $\nu(p)$ is also disjoint from $X$, and thus has positive curvature by construction. Consequently, $\rho$ is strictly convex on $T_{\nu(p)} \mathbf{S}^{n-1}$, which yields that $\rho_{\lambda}$ is also strictly convex. So $\partial K_{\lambda}$ has positive curvature on the complement of $X$. Now note that $\rho_{\lambda} \rightarrow \bar{\rho}$ as $\lambda \rightarrow 1$. Thus there exists a $\lambda_{0}<1$ such that $\operatorname{dist}\left(K_{\lambda_{0}}, \bar{K}\right) \leq \epsilon / 2$. The triangle inequality yields

$$
\operatorname{dist}\left(K_{\lambda_{0}}, P\right) \leq \operatorname{dist}\left(K_{\lambda_{0}}, \bar{K}\right)+\operatorname{dist}(\bar{K}, P) \leq \epsilon .
$$

Finally, suppose that $X$ is symmetric with respect to some rigid motion $m \in$ $O(n)$, i.e., $m(X)=X$. To make sure that $K_{\lambda_{0}}$ inherits the same symmetry, we may repeat the above procedure after replacing $\rho$ and $\bar{\rho}$ by

$$
\frac{1}{2}(\rho+\rho \circ m), \quad \text { and } \quad \frac{1}{2}(\bar{\rho}+\bar{\rho} \circ m),
$$

respectively. 


\section{ACKNOWLEDGMENTS}

The author thanks Ralph Howard for helpful comments, specially with regard to the proof of Proposition 2.1. Further, he is grateful to the editors and the referee for a detailed reading of this work, and suggestions for an improved exposition.

\section{REFERENCES}

[1] T. Bonnesen, and W. Fenchel, Theory of convex bodies, BCS Associates, Moscow, Idaho, 1987.

[2] S. S. Chern, and R. K. Lashof, On the total curvature of immersed manifolds, Amer. J. Math. 79 (1957), 306-318.

[3] W. Firey, Approximating convex bodies by algebraic ones. Arch. Math. (Basel) 25 (1974), 424-425.

[4] M. Ghomi, Strictly convex submanifolds and hypersurfaces of positive curvature, J. Differential Geom, 57 (2001) 239-271.

[5] — The problem of optimal smoothing for convex functions, Proc. Amer. Math. Soc., 130 (2002) 2255-2259.

[6] P. Gruber, Aspects of approximation of convex bodies, Handbook of convex geometry, Vol. A, 319-345, North-Holland, Amsterdam, 1993.

[7] P. Hammer, Approximation of convex surfaces by algebraic surfaces, Mathematika 10 (1963) 64-71.

[8] L. Helms, Brownian motion in a closed convex polygon with normal reflection, Ann. Acad. Sci. Fenn. Ser. A I Math. 17 (1992), no. 2, 199-209.

[9] H. Minkowski, Volumen und Oberfläche. Math. Ann., 57(1903), 447-495.

[10] R. Schneider, Smooth approximation of convex bodies, Rend. Circ. Mat. Palermo (2) 33 (1984), no. 3, 436-440.

[11] — Convex Bodies: The Brunn-Minkowski Theory, Encyclopedia of mathematics and its applications, v. 44, Cambridge University Press, Cambridge, UK, 1993.

[12] W. Weil, Einschachtelung konvexer Körper, Arch. Math., 26(1975), 666-9.

School of Mathematics, Georgia Institute of Technologies, Atlanta, GA 30332

E-mail address: ghomi@math.gatech.edu

$U R L$ : www.math.gatech.edu/ ghomi 